- Root canal treatment in children should only be prescribed after careful consideration of the patient, the existing dentition, and developing teeth.

- Isolation with rubber dam is just as important as with the permanent dentition.

- Paediatric endodontic treatment may be more directed towards pulpotomy rather than pulpectomy.

- All practitioners should be familiar with current guidelines on the treatment of the avulsed tooth.

\title{
Endodontic treatment for children
}

\section{P. Carrotte ${ }^{1}$}

Root canal treatment for children has particular difficulties and considerations. It must be planned in light of the remaining teeth, and the need for balancing or compensating extraction borne in mind. Diagnosis may be difficult, as may prolonged treatment under local anaesthesia and rubber dam. Vital pulpotomy techniques with formocresol and/or calcium hydroxide must be carefully executed in line with the UK National Guidelines. The treatment of the avulsed tooth has been the subject of much research, and practitioners should ensure that they are up-to-date with current treatment modalities.

\section{ENDODONTICS}

1. The modern concept of root canal treatment

2. Diagnosis and treatment planning

3. Treatment of endodontic emergencies

4. Morphology of the root canal system

5. Basic instruments and materials for root canal treatment

6. Rubber dam and access cavities

7. Preparing the root canal

8. Filling the root canal system

9. Calcium hydroxide, root resorption, endo-perio lesions

10. Endodontic treatment for children

11. Surgical endodontics

12. Endodontic problems

${ }^{*}$ Clinical Lecturer, Department of Adult Dental Care, Glasgow Dental Hospital and School, 378 Sauchiehall Street, Glasgow G2 3JZ

${ }^{*}$ Correspondence to: Peter Carrotte Email:p.carrotte@dental.gla.ac.uk

\section{Refereed Paper}

doi:10.1038/sj.bdj.4811946

๑ British Dental Journal 2005; 198: 9-15
Although the basic aims of endodontic therapy in children are the same as those in adults, ie the removal of infection and chronic inflammation and thus the relief of associated pain, there are particular difficulties and considerations. The pulpal tissue of primary teeth may become involved far earlier in the advancing carious lesion than in permanent teeth. Exposure may also occur far more frequently during cavity preparation due to the enamel and dentine being thinner than in the permanent tooth, and the pulp chamber, with its extended pulp horns, being relatively larger, as can be seen in the extracted tooth at Figure 1. Primary molar root canals are irregular and ribbon-like in shape. Periradicular lesions associated with infected primary molars are usually inter-radicular

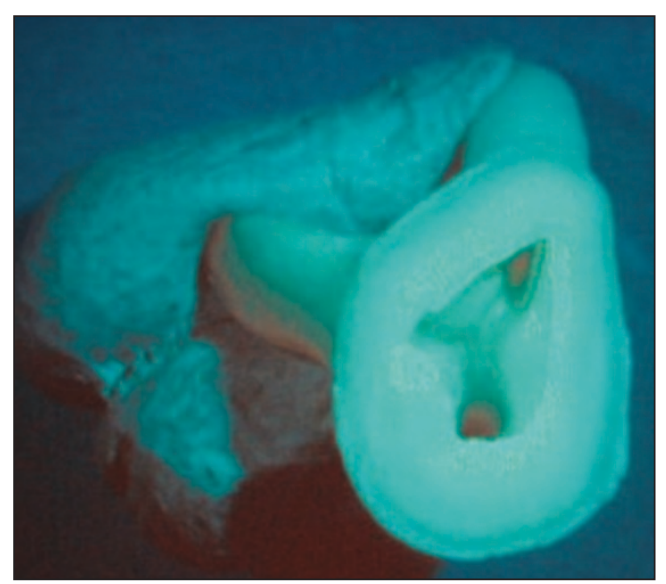

Fig. 1 An extracted deciduous molar showing the relatively large pulp chamber and root canals.
(Fig. 2) rather than periapical in site due to the presence of accessory canals in the thin floor of the pulp chamber.

As well as the problems associated with the primary dentition, endodontic treatment of permanent teeth in children may also present difficulties due to the incomplete root development and associated open apices.

\section{BALANCED EXTRACTIONS}

Primary teeth with pulpal exposure or pathology must always be treated, either by root canal treatment or by extraction. The maintenance of arch length is important for good masticatory function and the future eruption of the permanent dentition with optimal development of the occlusion. Whilst it is preferable to

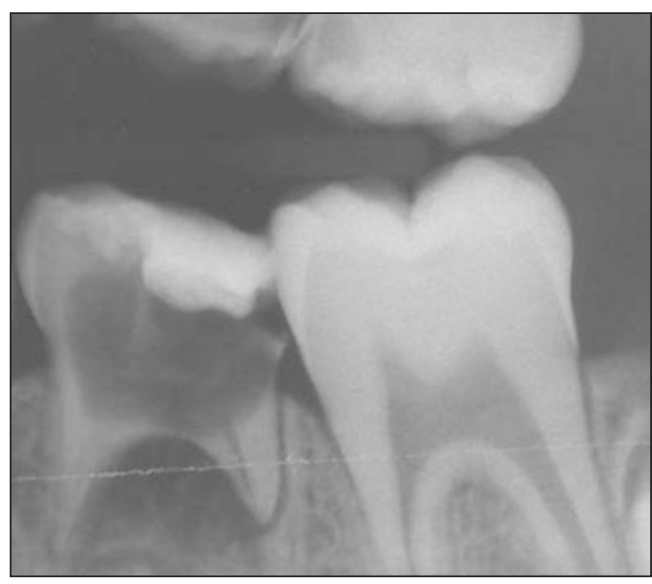

Fig. 2 A radiograph of a grossly carious lower second primary molar showing interadicular bone loss. 
Fig. 3 A dental panoramic tomograph taken as part of the assessment for extraction of deciduous molars, reveals the absence of permanent lower second premolars.

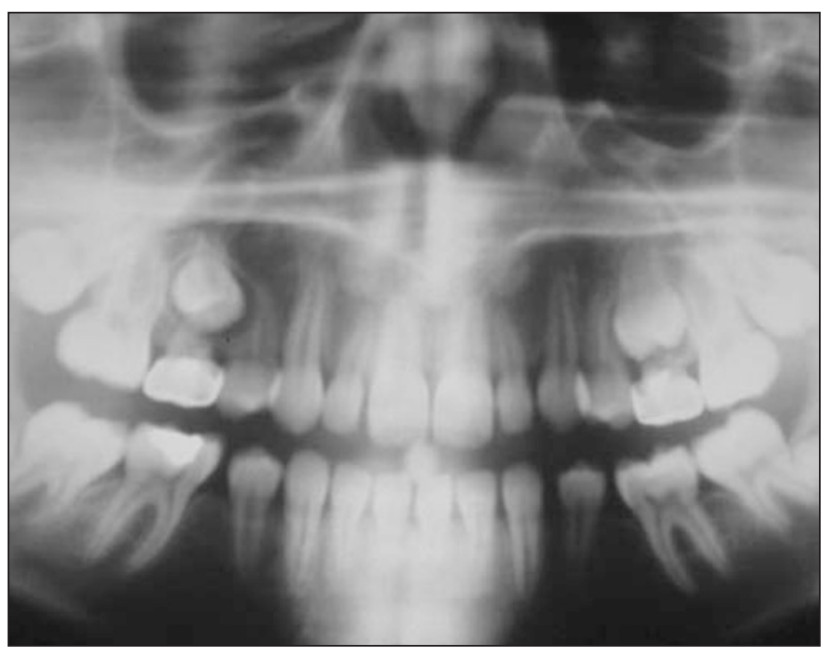

conserve a tooth rather than carry out an extraction, if this does become necessary, balanced extractions should always be kept in mind. A balanced extraction is the removal of a tooth from the opposite side of the same arch. A compensating extraction, removing a tooth from the opposing arch to the enforced extraction is more difficult to justify. ${ }^{1}$ Balanced extractions are rarely justified for primary incisors. The loss of a primary canine, however, may have a significant effect on the arch and balanced extractions should always be considered. When a primary molar has to be extracted it may be preferable to prevent drifting with a space maintainer than carry out balanced extractions.

Extractions should be avoided wherever possible in certain groups of children; ie those with bleeding disorders, or medical conditions such as diabetes where general anaesthesia is contraindicated. Primary teeth should also be retained where a radiograph reveals the lack of a permanent successor, as in Figure 3, where the patient may find pulp therapy less stressful than extraction, and in an already crowded dentition where tooth loss would lead to even further crowding of the permanent teeth.

\section{ENDODONTIC TREATMENT OF PRIMARY TEETH}

Endodontic treatment may be indicated far earlier when treating the primary dentition than in permanent teeth. Obviously, treatment is indicated when a patient presents with a pulpal necrosis, or symptoms of pulpitis. However, the distinction of reversible or irreversible pulpitis applied to the permanent teeth is not so relevant in the primary teeth; any sign or symptom of pulpitis indicates the need for pulp therapy. Current research and practice also suggests that pulp therapy will be necessary when a radiograph shows a carious lesion extending more than halfway through the dentine, or where the carious process has led to the loss of the marginal ridge.

However, there are important assessments to be made as to the patient's suitability for endodontic treatment. The general health of the patient should be checked to ensure that there are no contra-indications to endodontic therapy,

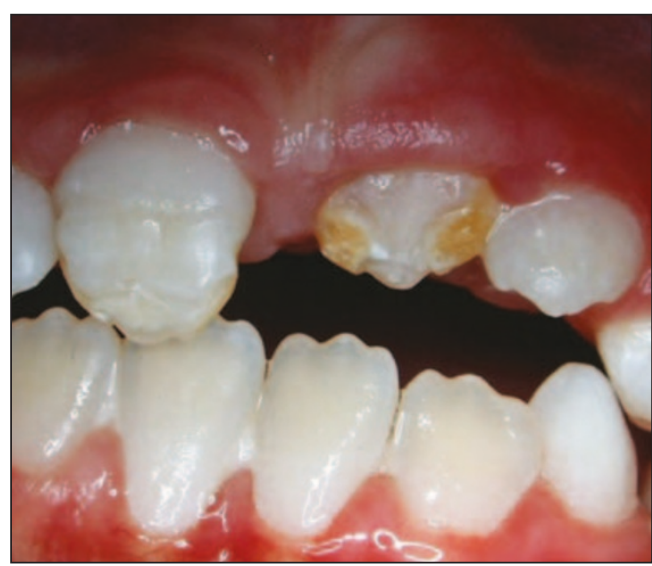

Fig. 4 Enamel hypoplasia of an upper permanent incisor following infection of the primary predecessor.

such as those with congenital heart disease, or patients who are immunocompromised. The attitude of the parent to treatment and the child's ability to cooperate during the more lengthy procedures require careful evaluation. The overall dental health of the child, with particular reference to the caries experience, must be taken into account when making a treatment plan. In a poorly cared for dentition requiring multiple treatments, the complex conservation of one tooth in the presence of a number of comparable teeth of doubtful prognosis is poor paediatric dentistry and should be avoided. In addition, root canal treatment should be avoided in grossly decayed teeth which may be unrestorable even after pulp therapy; in teeth where caries has penetrated the floor of the pulp chamber; in teeth with advanced root resorption, or those close to exfoliation.

An additional problem is the close relationship of the roots of the primary teeth to the developing permanent successor. During exfoliation, the roots of the former resorb, necessitating the use of a resorbable paste in endodontic treatment. It is also important to remember that trauma to, or infection of, a primary tooth, may result in damage to the permanent tooth. This may vary from enamel hypomineralisation and hypoplasia to, more rarely, the delayed or arrested development of the tooth germ (Fig. 4).

\section{Diagnosis}

The reaction of pulp tissue in primary teeth to deep caries differs from that seen in the permanent dentition and is characterised by the rapid spread of inflammatory changes throughout the coronal portion of the tooth. These pathological changes become irreversible and, if left untreated, will involve the radicular tissue. There may be few, if any, clinical symptoms in the early stages to indicate the extent of tissue damage. Pain may only occur after involvement of the periradicular tissues in the spread of infection.

Children are often unable to give accurate details of their symptoms, and the responses to clinical tests may be unreliable. Difficulties are frequently experienced in ascertaining the condition of the pulp from clinical findings. 


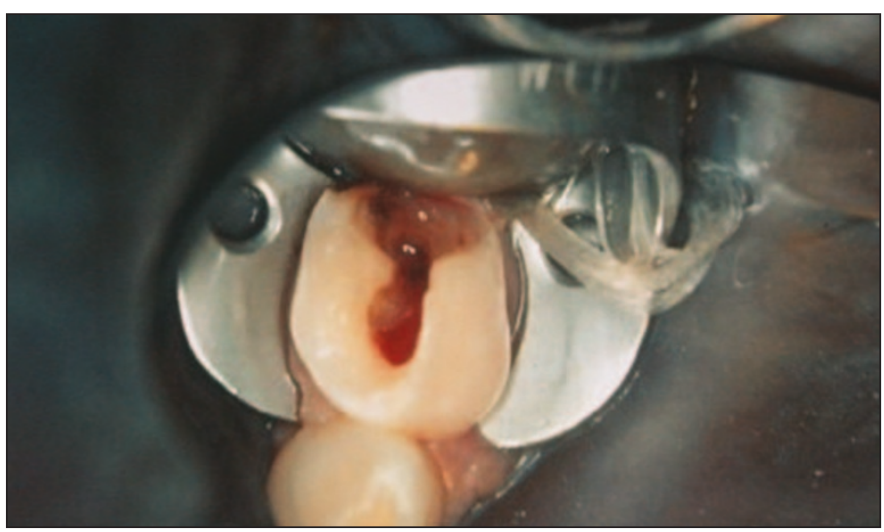

Fig, 5 A deciduous molar with a deep carious lesion has been isolated prior to commencing endodontic therapy.

Radiographs, which are essential prior to the commencement of treatment, may give little information of early pathological changes.

\section{Before commencing treatment}

The majority of the following restorative procedures will require adequate local anaesthesia. In accordance with the biological principles established throughout this text, adequate isolation will also be necessary to prevent salivary contamination. A rubber dam should be placed, and isolation completed with cotton wool rolls and saliva ejector as seen in Figure 5.

\section{Indirect pulp capping}

The aim of this treatment is to maintain the vitality of the pulp in a deep carious lesion, when there is no direct pulpal involvement. All the carious dentine must be removed, and a thin layer of sound, non-carious dentine must remain. A lining of setting calcium hydroxide is placed, which stimulates the formation of secondary dentine. The tooth is restored over the dressing with a permanent restorative material.

It has been suggested that other medicaments may be used for indirect pulp caps, for example antibiotic pastes and anti-inflammatory drugs, but although some success has been reported, pulp necrosis and abscess formation often result without symptoms. As with the permanent dentition, research is presently focussing on the use of adhesive materials and bonding agents for indirect pulp capping. The long-term results of these long-term clinical trials are awaited.

It should be noted that one technique for indirect pulp capping, which was described in the past, is no longer recommended. This was where deep caries was carefully excavated, avoiding pulpal exposure, and the deeper layers of softened dentine dressed with a calcium hydroxidecontaining cement and a long-term temporary dressing. After a period of 6-8 weeks the tooth, which should have been symptomless, was reopened, and the arrested carious lesion examined. The success of this treatment was found to be less predictable and symptoms frequently developed. It is now recommended that all caries be removed, and if a pulpal exposure is found then either a direct pulp cap or a form of pulpotomy is used.

\section{Direct pulp capping}

This treatment is only recommended when a small traumatic exposure occurs, during cavity preparation of a vital non-infected pulp. ${ }^{2}$ A calcium hydroxide dressing is placed directly over the pulp, followed by a lining and restoration, and the whole technique is carried out using local anaesthesia and with adequate isolation from salivary contamination. It has been suggested that the high cellular content of primary pulp tissue may be responsible for the failure of direct pulp capping in primary teeth. ${ }^{3}$ Undifferentiated mesenchymal cells may differentiate into osteoclastic cells in response to either the caries or direct pulp capping which leads to internal resorption. It is also suggested that exposures on axial walls have a poor prognosis as the pulp coronal to the exposure may be deprived of its blood supply and undergo necrosis.

\section{Vital pulpotomy techniques}

These techniques involve the removal of inflamed coronal pulp tissue and the application of a dressing to the radicular pulp in an attempt to either promote healing of, or fix, the upper portions, and to preserve the vitality of the apical tissue. Because of the difficulties involved in diagnosing the condition of the pulp tissue histologically before the commencement of treatment, careful assessment must be made at each stage of the procedure. Whenever the haemorrhage from the radicular pulp stumps is profuse and uncontrolled, the assumption is made that the inflammatory process has extended into the radicular tissue, and the therapy modified accordingly. There are three pulpotomy techniques.

\section{Vital formocresol pulpotomy}

The treatment is carried out using local anaesthesia and adequate isolation. Following cavity preparation in the normal manner, the deep caries is removed and the coronal pulp chamber opened, such that there is no overhanging dentine inhibiting the complete removal of the pulp tissue. The coronal tissue is removed using a large excavator or sterile rosehead bur. If a high-speed diamond bur is used it should be cooled with sterile water or saline. Sterile cotton wool is applied to the radicular pulp tissue to achieve haemostasis. A small pledget of cotton wool is dipped in a 1:5 dilution of Buckley's formocresol (Table 1) and 


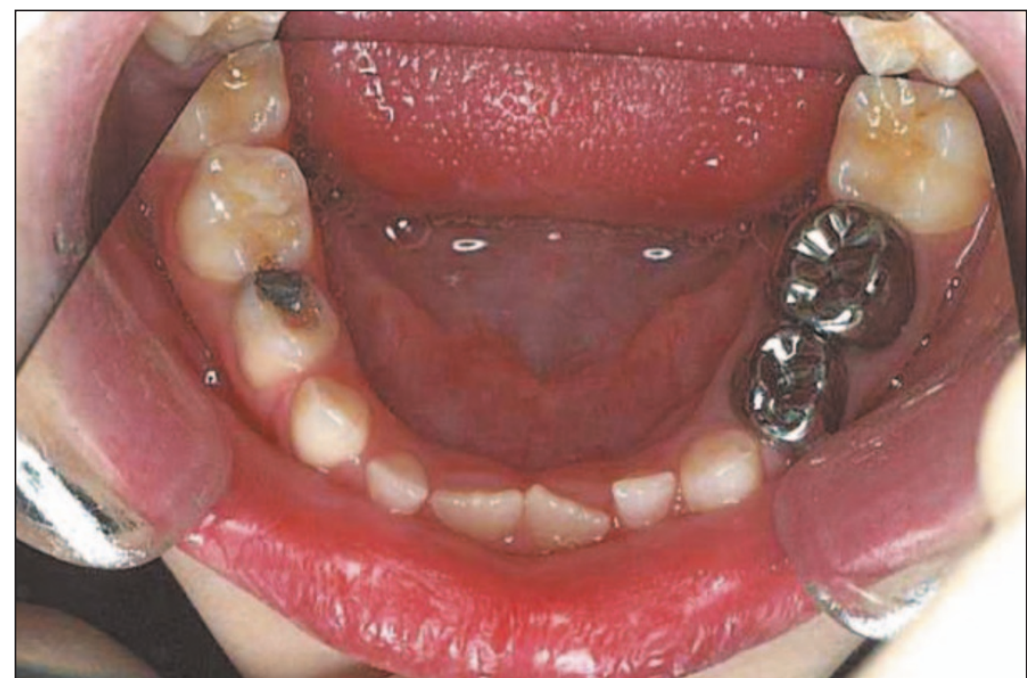

\begin{tabular}{lc} 
Table 1 Buckley's formocres \\
\hline Tricresol & $35 \%$ \\
Formaldehyde & $19 \%$ \\
Glycerol & $15 \%$ \\
Water & $31 \%$
\end{tabular}

squeezed to remove excess liquid. It is placed over the radicular pulp stump for 5 minutes in order to fix the inflamed tissue and bacteria and thus allow healing of the unaffected pulp. If the haemorrhage has completely stopped, a layer of zinc oxide-eugenol or glass-ionomer cement is applied, and the tooth restored, preferably with a preformed stainless steel crown to prevent subsequent fracture of the weakened tooth (Fig. 6).

Other materials have been considered as an alternative to formocresol. ${ }^{4}$ Concerns about the safety of formocresol led to investigations of pulpotomies employing a 2\% glutaraldehyde solution as an alternative dressing, but research has shown a lower clinical success rate than with formocresol. Concern about hypersensitivity to and handling of glutaraldehyde have largely led to its abandonment as a treatment alternative.

Recent work by Waterhouse et al. has shown that very favourable results have been achieved with calcium hydroxide when it has been applied in carefully controlled circumstances. ${ }^{5}$ Following haemostasis, calcium hydroxide powder was delivered to the pulp chamber using a small, sterile, endodontic amalgam carrier. The powder is condensed over the pulp stumps with an amalgam condensor and small pledgets of cotton wool. Failure of this technique is explained by the presence of an extra-pulpal clot separating the calcium hydroxide from the pulpal tissue and thus impairing healing. ${ }^{3}$

Both the calcium content and alkaline properties of the dressing are important to achieve healing. An initial layer of necrotic tissue develops, which becomes associated with an inflammatory reaction. Subsequently, a matrix forms and mineralises to become a hard tissue barrier of dentine-like material.

\begin{tabular}{lc} 
Table 2 Paraformaldehyde devitalising paste \\
\hline Paraformaldehyde & $1.00 \mathrm{~g}$ \\
Carbowax 1500 & $1.30 \mathrm{~g}$ \\
Lignocaine & $0.06 \mathrm{~g}$ \\
Propylene glycol & $0.5 \mathrm{ml}$ \\
Carmine & $10 \mathrm{mg}$
\end{tabular}

\section{Devitalisation pulpotomy}

This is a two-stage procedure, used when local anaesthesia cannot be obtained to permit extirpation of the pulp, or when haemorrhage is uncontrolled before or following the application of formocresol. This technique mummifies and fixes the coronal pulp tissue, whilst the major part of the radicular pulp remains vital, but it carries a lower success rate. ${ }^{6}$

If the tooth is not anaesthetised, cavity preparation is carried out as far as possible and access is gained to the pulpal exposure. A small amount of paraformaldehyde devitalising paste (Table 2) on a pledget of cotton wool is applied to the exposed pulp tissue. Formaldehyde vapour liberated from the dressing permeates through the pulpal space, producing fixation of the tissues. A soft layer of zinc oxide-eugenol temporary dressing is then placed, without applying pressure, to seal the medicament in position. The child and parent must be warned of possible discomfort, for which analgesics are recommended. After one to two weeks the tooth is checked for signs and symptoms. The devitalised coronal pulp may now be removed, without the need for local anaesthesia. A hard setting layer of zinc oxide-eugenol, which may be mixed with formocresol, is then placed over the radicular stumps and the tooth restored. If some vital tissue remains in the coronal pulp chamber, a further dressing of paraformaldehyde paste is required.

\section{Non-vital pulpotomy}

This technique has been advocated where there is irreversible change in the radicular pulp, or where the pulp is completely non-vital, but where pulpectomy and root canal treatment is considered impractical. The little clinical evidence 
available suggests a limited prognosis of approximately 50\%. At the first visit the necrotic pulp contents are removed as before, and, using small excavators, as much as possible of the radicular tissue. Beechwood creosote solution (Table 3) on a cotton pledget is sealed into the cavity with a zinc oxide-eugenol dressing.

One to two weeks later the tooth is checked for signs and symptoms. If there is evidence of infection (sinus, pain, swelling or mobility) a further beechwood creosote dressing should be placed. If, however, symptoms have resolved, the tooth may be restored as with the previous pulpotomy techniques.

\section{Pulpectomy}

Pulpectomy is indicated where the pulp is either non-vital or irreversibly inflamed. Although the technique is often considered difficult because of the complexity of the root canals in primary molars, clinical studies have shown a reasonable prognosis. ${ }^{7}$ The cavity preparation and removal of the necrotic coronal pulp is carried out as previously described. If the radicular pulp is necrotic, a two-stage procedure is required, but if it is found to be irreversibly inflamed a one-stage technique may be undertaken.

\section{One-stage technique}

The root canals are identified and instrumented to the working length estimated from a pre-operative radiograph. After drying the canals with paper points, formocresol is applied for up to 5 minutes. The root canals are then filled with a thin mix of zinc oxide-eugenol, using a rotary paste filler, and the restoration of the tooth is completed.

\section{Two-stage technique}

Here the root canals are again cleaned, shaped and irrigated to remove all necrotic debris. A pledget of cotton wool moistened with either formocresol or beechwood creosote is sealed in the pulp chamber with a rigid zinc oxideeugenol dressing for one week. At the subsequent visit the tooth should be symptom-free, firm, without a discharging sinus. (If not, a second application of beechwood creosote is required.) If the tooth is found to be symptomless, a dressing of zinc oxide-eugenol, with or without the addition of formocresol, is packed into the base of the chamber and the tooth finally restored.

The preceding techniques are reviewed in the UK National Guidelines. ${ }^{8}$

\section{Review}

Following any form of endodontic treatment, regular clinical and radiographic reviews must be made of the tooth involved and its successor. If rarefaction of the bone in the furcation area is seen, further pulpectomy may be possible, but extraction is probably indicated. Radiographs should also be checked for evidence of internal resorption, which may occur in limited areas in formocresol pulpotomies, but may be more extensive following the use of calcium hydroxide. It may progress to cause perforation of the

\begin{tabular}{lr} 
Table 3 Beechwood creosote \\
\hline O-Methoxy phenol (Guaicol) & $47 \%$ \\
P-Methoxy phenol & $26 \%$ \\
2-Methoxy, 4 methyl phenol (Cresol) & $13 \%$ \\
M-Methoxy phenol & $7 \%$ \\
Other & $7 \%$
\end{tabular}

root. Inflammatory follicular cysts ${ }^{9}$ may develop, which necessitate the removal of the primary tooth and marsupialisation of the cyst to allow the permanent tooth to erupt.

\section{PERMANENT DENTITION}

\section{Immature permanent incisors}

Although one in five children will suffer trauma to their developing permanent incisors, only about $6 \%$ of these will subsequently become non-vital and require endodontic treatment. The correct initial diagnosis of such traumatised teeth, based on signs and symptoms, radiographic examination and sensibility testing, is therefore very important. Laser Doppler flowmetry has shown that traumatised immature teeth with open apices may have a vital pulp even in the absence of a response to conventional sensibility testing. If there is any uncertainty about the vitality of the pulp, root canal treatment should be deferred and the tooth kept under regular review.

If, however, endodontic treatment of an immature permanent tooth with an open apex is indicated, a root-end closure technique is necessary to form a calcific barrier against which the obturation may eventually be compacted without extruding material into the periradicular tissues (Fig. 7).

The tooth should be isolated with rubber dam, and the pulp chamber accessed. Local anaesthesia is usually given as some vital tissue may still be encountered during pulp extirpation. In severe cases an intracanal steroid dressing, such as Ledermix, may be required for one week. Canal preparation is carried out with files to approximately 1-2 $\mathrm{mm}$ short of the working length, estimated from the pre-operative radiograph and confirmed during treatment. Copious irrigation with a sodium hypochlorite solution is necessary to remove all necrotic debris. The root canal should be dried with paper points, and then filled to the apex with calcium hydroxide paste, compressed with large paper points and/or cotton pledgets. The access cavity should be sealed with a long-term temporary dressing, such as glass-ionomer cement.

After one month, the dressing is carefully removed with copious irrigation, and the dried canal refilled with calcium hydroxide paste. After a further three to six months the tooth is opened again and a large paper point used at working length to feel for a calcific barrier. The paper point is gently inserted into the clean, dry canal. At the estimated working length either the point will remain dry, tap against a hard barrier, 
Fig. 7 a) An immature tooth with a non-vital pulp has been filled with calcium hydroxide b).

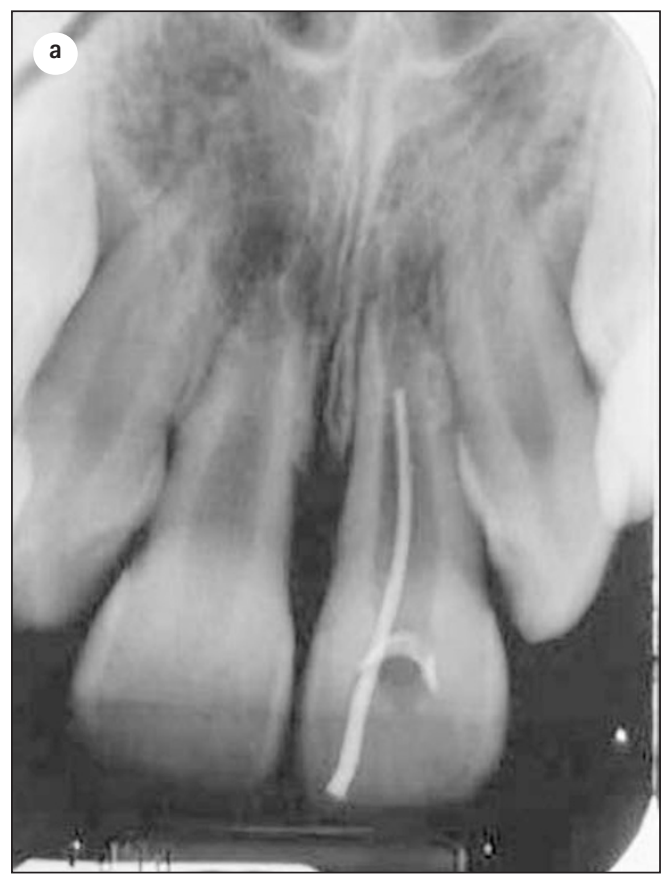

with no sensation to the patient indicating closure, or will press against soft granulation tissue which the patient will feel. The average time taken for closure is 6 months.

If no barrier is detected, the calcium hydroxide is replaced. If the open apex is found to be completely closed, the canal may be obturated with gutta-percha and sealer. Closure of an open apex may be anticipated in over 90\% of cases treated by this technique, with a 4-year prognosis of $85 \% .{ }^{10}$ Obturation may then be completed by one of several methods. Conventional cold lateral compaction may be used, perhaps inverting a large gutta-percha point to obtain a good apical tug-back. A custom gutta-percha point may be made by rolling several GP points together after softening in solvent or gentle heat, and repeated fitting to the canal, carefully marking the orientation at insertion. However, injectable thermo-plasticised gutta-percha may be the most suitable obturation medium.

\section{First permanent molar}

The first permanent molar may, soon after eruption, show extensive caries, sometimes associated with hypoplasia. Consideration must be given to the age of the patient and the dental development, the occlusion and possible need for orthodontic treatment, as well as the long-term restorative prognosis of the tooth and the patient's ability to tolerate involved treatment over a long period. Where necessary, planned extractions should be considered. The primary aim of conservation is to ensure that root growth continues with completion of apical formation, so that definitive endodontic treatment, if required, may be carried out at a later stage.

The vitality of the tooth must be assessed and radiographs should be available, showing the extent of carious involvement and the state of the periapical tissues. It is essential that a local anaesthetic is administered and salivary control

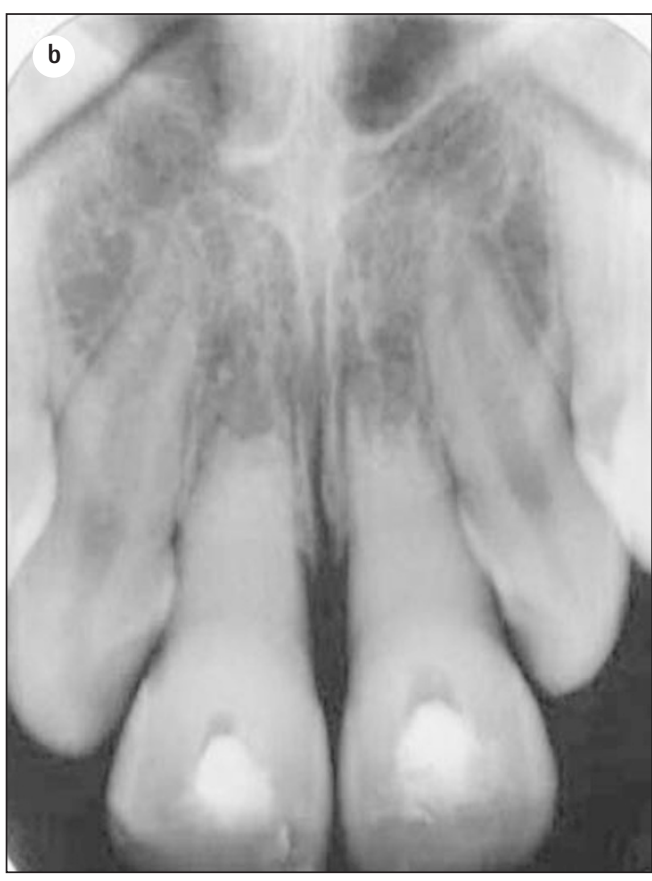

achieved by adequate isolation. Caries should be excavated and the tooth treated in accordance with conventional protocols. If a small exposure of a vital tooth occurs, either accidentally during cavity preparation or because of caries, and the surrounding tissue is healthy, a direct pulp cap with calcium hydroxide cement may be applied. A lining of glass-ionomer cement is then placed to seal the dentine tubules prior to the definitive restoration. If amalgam is used, a dentine bonding system should be considered to ensure complete sealing of the restoration.

If the exposure is large and the vitality of the radicular pulp is to be maintained to allow for root development, a pulpotomy may be carried out. Following the opening of the coronal pulp chamber and the removal of the pulp tissue, the area is irrigated and dried. Haemostasis of the radicular pulp should be observed prior to the application of calcium hydroxide cement or paste, and the provision of a permanent restoration. A calcific barrier should develop adjacent to the dressing, and root development continue in the presence of healthy pulp tissue.

If the pulp of a young, permanent molar is found to be non-vital, endodontic treatment should be undertaken only after careful assessment of the developing occlusion, the condition of the comparable teeth, the patient's ability to cooperate and the long-term prognosis of the tooth. If pulpal necrosis occurs prior to the complete development of the apex, the objective of treatment, as described earlier, is to encourage further deposition of calcified tissue in the apical region. Thorough preparation of the root canals is carried out, avoiding damage to the apical tissues and cells of Hertwig's root sheath. Calcium hydroxide is then applied as previously described. Definitive endodontic treatment is carried out when an apical barrier has formed and the tooth is then permanently restored. If symptoms arise in fully developed, young, 
permanent teeth, conventional orthograde rootfilling with gutta-percha and sealer is indicated.

\section{Avulsed permanent teeth}

In the emergency management of an avulsed permanent tooth, time is of the essence. The long-term prognosis begins to deteriorate after only 15 minutes. ${ }^{11}$ Most cases initially present with a telephone call. Where possible, reimplantation should be immediate, following rinsing if necessary in either milk (preferably) or tap water. The tooth should be held in place by biting gently on a soft cloth until splinting is possible by the dentist. If the person attending the accident is not prepared to re-implant the tooth, it should be stored in milk, normal saline or saliva (in the buccal sulcus) during the journey to the dental surgery.

Avoiding unnecessary delay, and keeping the tooth in the transport solution to prevent drying of the periodontal fibres, a thorough medical, dental and accident history should be taken and recorded. Local anaesthesia may be necessary to permit manipulation of the alveolar bone, and to enable gentle syringing of the socket with saline to remove any blood clot. The tooth, handled only by the crown, should be carefully inserted into the socket. Root canal treatment should NOT be commenced before re-implantation.

A non-rigid splint should be applied for 7-10 days, using acid-etched resin with a soft arch wire. The patient should be advised to avoid biting on the splinted tooth, take a soft diet, and maintain good oral hygiene with careful brushing and a chlorhexidine mouth-rinse. Systemic antibiotics may be indicated for medically compromised patients. The patient's tetanus status must be checked and a booster given by a medical practitioner if necessary. A review appointment should be made in two days to verify the splint, and modify it if necessary.

In very young patients where the tooth has a wide-open apex and was out of the mouth for only a short period there is a possibility of revascularisation of the pulp. The tooth should be kept under almost weekly review, and if any clinical signs of non-vitality develop, such as tenderness, discoloration, swelling or sinus formation, endodontic treatment should be commenced immediately. Endodontic treatment should be commenced on all other avulsed teeth whilst the splint is in place. A long-term calcium hydroxide dressing should be sealed in place with a glass-ionomer restoration for at least 6 months prior to verification of an apical barrier and obturation as described earlier.

Replanted teeth should be regularly reviewed for at least 2-3 years, checking for inflammatory resorption, replacement resorption, ankylosis, infra-occlusion and discoloration. The adjacent teeth should also be reviewed. Resorption may commence within weeks of the injury.

Finally, it should be realised that there are some situations where replantation is not appropriate. For example:

- If the patient has other serious injuries, which should be given priority.

- If the patient has an at-risk medical history.

- Where the extra-oral time is very prolonged, the prognosis is very poor, particularly in teeth with short roots and wide apices.

- Primary teeth should not be replanted due to the possibility of damage to the permanent replacement.

Figures 1, 2, 3, 5, 6 and 7 have been reproduced by kind permission of Dr M-T Hosey, Children's Department. Glasgow University.

Figure 4 is reproduced by kind permission of Professor $R R$ Welbury.

1. BSPD and IAPD. UK national guidelines in paediatric dentistry. Int J Paediatr Dent 2002; 12: 151-153.

2. Kopel H M. Considerations for the direct pulp capping procedure in primary teeth: A review of the literature. Paediatr Dent 1992; 59: 141-149.

3. Gould A, Johnstone S, Smith P. Pulp Therapy techniques for the deciduous dentition. (Compact Disk) London: King's College, 1999.

4. Waterhouse P J. Formocresol and alternative primary molar pulpotomy medicaments: a review. Endod Dent Traumatol 1991; 11: 157-162.

5. Waterhouse P J, Nunn J H, Whitworth J M. An investigation of the relative efficacy of Buckley's Formocresol and Calcium Hydroxide in primary molar vital pulp therapy. Br Dent J 2000; 188: 32-36.

6. Coll J A, Sadrian R. Predicting pulpotomy success and its relationship to exfoliation and succedanesus dentition. Paediatr Dent 1996; 18: 57-63.

7. Barr E S, Flaitz C M, Hicks M J. A retrospective radiographic evaluation of primary molar pulpectomies. Paediatr Dent 2000; 13: 4-9.

8. Llewelyn D R. UK national guidelines in paediatric dentistry. Int J Paediatr Dent 2000; 10: 248-252.

9. Shaw W, Smith D M, Hill F J. Inflammatory follicular cysts. J Dent Child 1980; 47: 97-101.

10. Mackie I C, Worthington H V, Hill F J. A follow up study of incisor teeth which have been treated by apical closure and root filling. Br Dent J 1993; 175: 99-101.

11. Andersson L, Bodin I. Avulsed human teeth replanted within 15 minutes - a long term clinical follow-up study. Endod Dent Traumatol 1990; 6: 37-42. 